\title{
Tecnura
}

\section{Producción de biodiésel a partir del aceite extraído de almendra del corozo Bactris guineensis asistida mediante ultrasonido de sonda directa}

\section{Biodiesel Production From Almond Oil Extracted From the Corozo Bactris Guineensis Assisted by Direct Probe Ultrasound}

\author{
Juan Martín Madrid De la Rosa (iD) 1 , Dary Mendoza Meza (iD) 2, Miriam Fontalvo Gómez (iD)
}

Fecha de Recepción: 14 de Diciembre de 2020

Fecha de Aceptación: 01 de Abril de 2021

Cómo citar: Madrid De la Rosa., J.M. Mendoza-Meza., D. y Fontalvo-Gómez., M. (2021). Producción de biodiesel a partir del aceite extraído de almendra del corozo Bactris guineensis asistida mediante ultrasonido de sonda directa. Tecnura, 25(69), 51-75. https:/ / doi.org/10.14483/22487638.15809

\section{Resumen}

Contexto: Teniendo en cuenta que el corozo (Bactris guineensis) es una fruta exótica del Caribe colombiano utilizada localmente en la industria de los alimentos y que su almendra posee alto contenido oleaginoso $(39,43 \% \pm 0,49)$, el presente trabajo busca abordar el aprovechamiento del aceite de la almendra del corozo como alternativa en la obtención de biodiesel de ultrasonido de sonda directa como medio de agitación.

Métodos: Los ensayos se llevaron a cabo bajo diferentes valores de relación molar alcohol: aceite, concentración de catalizador, amplitud de ultrasonido y tiempo. Para ello se utilizó un diseño central compuesto y se usó la variable de respuesta "rendimiento de reacción" para determinar las condiciones óptimas para la obtención de biodiésel.

Resultados: Se obtuvo un rendimiento máximo de reacción del 81,05\% una relación molar alcohol: aceite de 6:1; concentración de catalizador de 0,70\%; amplitud de la sonda de ultrasonido del $20 \%$ y tiempo de reacción de 15 minutos.

Conclusiones: Se demostró que el producto final cumple con la mayoría de las propiedades señaladas por la norma técnica colombiana (NTC 5444). La implementación del ultrasonido de sonda directa permitió obtener altos rendimientos con tiempos de reacción inferiores a los reportados con los métodos convencionales de agitación, lo cual representa una oportunidad de reducir los gastos energéticos asociados con la producción del biocombustible. Adicionalmente, la diversificación de las materias primas para la fabricación de biodiésel con el aprovechamiento de los ácidos grasos extraídos de la biomasa residual de B. guineensis, representa una oportunidad para el impulso de la economía regional.

Palabras clave: aceite de corozo, transesterificación, ultrasonido, biocombustibles, desechos alimenticios, energía alternativa.

\footnotetext{
${ }^{1}$ Ingeniero químico. Joven investigador de Colciencias en Universidad del Atlántico. Barranquilla, Colombia. Email: jmadrid@mail.uniatlantico.edu.co

${ }^{2}$ Química farmacéutica, doctora en Biotecnología. Docente de la Universidad del Atlántico. Barranquilla, Colombia. Pharmaceutical Chemist, PhD in Biotechnology. Professor at the Universidad del Atlántico. Barranquilla, Colombia. Email: darymendoza@mail.uniatlantico.edu.co

${ }^{3}$ Química farmacéutica, doctora en Química. Docente de la Universidad del Atlántico. Barranquilla, Colombia. Email: miriamfontalvo@mail.uniatlantico.edu.co
} 
Producción de biodiesel a partir del aceite extraído de almendra del corozo Bactris guineensis asistida mediante ultrasonido de sonda directa

Madrid De la Rosa., J.M. Mendoza-Meza., D. y Fontalvo-Gómez., M.

\begin{abstract}
Context: Corozo (Bactris guineensis) is an exotic fruit from the Colombian Caribbean. It is used locally in the food industry. Corozo has high oleaginous content $(39,43 \% \pm 0,49)$ and it could be an option for the oil industry in Colombia. This study shows the use of Corozo oil as an alternative for biodiesel production using ultrasound with a direct probe as the agitation mechanism.

Method: The experiments were carried out under different values to alcohol: oil molar ratio, catalyst concentration, ultrasound intensity, and reaction time. For this purpose, a central compound design was used as the response variable "reaction yield" was used to set the best conditions in biodiesel production.

Results: A maximum reaction yield (81, $05 \%$ ) was obtained using alcohol: oil molar ratio of 6:1; catalyst concentration of $0,70 \%$; the amplitude of the ultrasound probe of $20 \%$ and the reaction time of 15 minutes.

Conclusions: It was found biodiesel fulfills most of the properties set by the Colombian technical standard (NTC 5444). Direct probe ultrasound shows high yields with times lower than those reported with conventional methods which represent an opportunity to reduce the energy cost associated with biofuel production. Additionally, the variation of raw materials for the manufacturing of biodiesel through the use of oils from the residual biomass of B. guineensis represents an opportunity to boost the regional economy.
\end{abstract}

Keywords: Corozo oil, transesterification, ultrasound, biofuels, food waste, alternative energy.

\title{
Tabla de Contenidos
}

Página

Introducción

Metodología

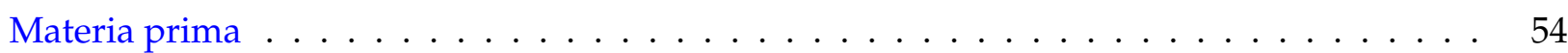

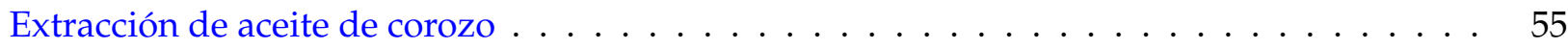

Refinación y caracterización . . . . . . . . . . . . . . . . . . 55

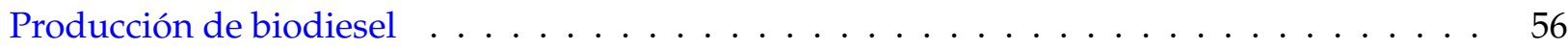

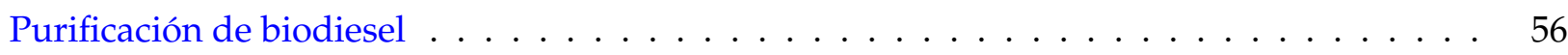

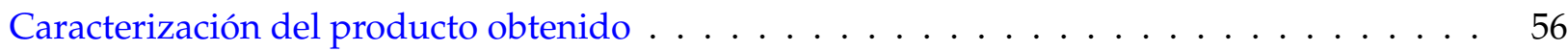

Caracterización cualitativa del biodiesel . . . . . . . . . . . . . 56

Caracterización fisicoquímica del biodiesel . . . . . . . . . . . . . . 58

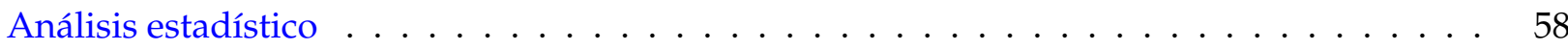

$\begin{array}{lc}\text { Resultados } & 58\end{array}$

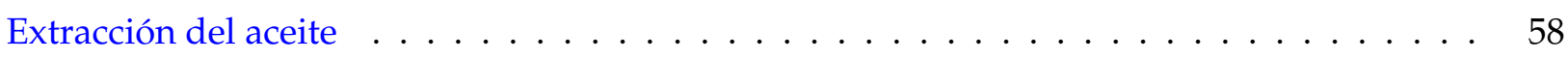

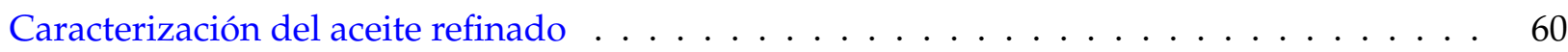

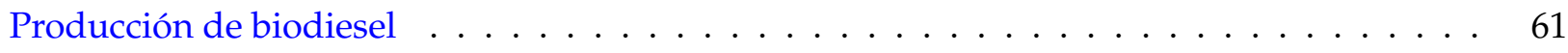

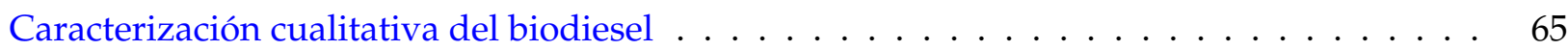


Producción de biodiesel a partir del aceite extraído de almendra del corozo Bactris guineensis asistida mediante ultrasonido de sonda directa

Madrid De la Rosa., J.M. Mendoza-Meza., D. y Fontalvo-Gómez., M.

Caracterización fisicoquímica del biodiesel $\ldots \ldots \ldots \ldots \ldots \ldots$

$\begin{array}{lr}\text { Conclusiones } & 68\end{array}$

$\begin{array}{lr}\text { Financiamiento } & 68\end{array}$

$\begin{array}{lr}\text { Agradecimientos } & 69\end{array}$

$\begin{array}{lr}\text { Referencias } & 69\end{array}$

\section{Introducción}

La propagación de gases nocivos en la atmósfera, producto del uso de combustibles fósiles, es un tema, que involucra a la población, la industria y la sociedad científica de cada país. Hoy en día, existe un gran compromiso por generar alternativas energéticas que ayuden a mitigar la necesidad de combustibles fósiles, con miras a reducir la carga de contaminación (Gaurav et al., 2017, Flórez et al., 2016). Los biocombustibles producidos a partir de materias primas renovables no convencionales surgieron desde hace algunos años, como una opción para mermar la presencia de gases invernadero en el ambiente (Esteves et al., 2018, Rassoulinejad-Mousavi et al., 2018, Andrade et al., 2016). El biodiesel es un combustible renovable (Liu et al., 2018) cuyo uso reduce la emisión $\mathrm{CO}_{2}$, $\mathrm{CO}$ (Mejía y Cuty, 2019) y otros contaminantes (Thapa, Indrawan, y Bhoi, 2018); y que consiste principalmente en ésteres metílicos de ácidos grasos (EMAG) derivados de aceites vegetales por procesos de transesterificación o esterificación (Yu et al., 2017).

En Colombia, el componente primario utilizado para la manufactura industrial de biodiesel es el aceite de palma africana (Elaeis guineensis). Se estima que aproximadamente el 60 \% de la producción nacional de este aceite se destina al sector alimenticio, mientras que el $40 \%$ se utiliza para la producción de biocombustibles (Granados y Valencia, 2018). Este panorama ha generado una competencia entre la seguridad alimentaria y el sector energético (Martínez, Arango, y Giraldo, 2019), planteándose la necesidad de buscar materiales alternativos para la obtención de biodiesel; una opción es el aprovechamiento de la biomasa de plantas provenientes de los residuos de la agroindustria (Núñez, 2012, Romero et al., 2016).

Por su ubicación geográfica, Colombia presenta una gran diversidad en plantas oleaginosas que podrían constituirse en muy buenas opciones para la industria de los aceites (Esquinas et al., 2018, Teixeira et al., 2018). Este recurso biológico ofrece una oportunidad magnífica para la exploración y desarrollo de biocombustibles a partir de materiales no convencionales que, a su vez, no son aptas para el consumo humano o que son consideradas material de desecho. Según (Durán et al., 2018), Colombia produce cerca de 12 millones de toneladas de desechos; $53 \%$ proceden de residuos agroindustriales como frutas y cáscaras; mucha de esta biomasa residual posee alto contenido en aceite, razón por la 
cual puede ser aprovechada por el sector energético (Amaringo y Hormaza, 2018) para la elaboración de productos de valor, entre ellos el biodiesel (Rojas, Flórez, y López, 2019).

El corozo (Bactris guineensis (L.) H.E. Moore 1963; familia: Arecaceae) es una palma que se cultiva en el Caribe Colombiano. Su fruto es utilizado en las industrias fruteras para la producción de jugos y mermeladas (Bernal y Galeano, 2013), proceso en el cual se genera una cantidad considerable de biomasa residual que es desaprovechada. Esta biomasa se compone principalmente de la cáscara y de la almendra húmeda de corozo; esta última posee un contenido importante de triacilglicéridos (TAG), entre 39 - 40 \% (Polo et al., 2018). El alto contenido de TAG es un parámetro determinante en la selección de materiales potenciales para la obtención de energía renovable puesto que proporciona información acerca de la productividad de aceite por hectárea sembrada (Durišić-Mladenović et al., 2018).

En el presente estudio se propuso utilizar los residuos del corozo provenientes de algunas fruteras locales, para la obtención de biodiesel por transesterificación convencional, implementando la técnica de mezclado con ultrasonido de sonda. Estudios previos demuestran que este sistema de agitación acelera la reacción de transesterificación debido al aumento de colisiones entre los reactivos como resultado de vibraciones y turbulencias derivadas de un fenómeno conocido como cavitación (Patle et al., 2018), lo que supone una reducción en el tiempo de reacción y en consecuencia un menor gasto energético. La transesterificación asistida por ultrasonido ha sido usada en la transformación de aceites vegetales comestibles (e.g. aceite de canola, maíz, palma, soya y girasol) a biodiesel, obteniéndose rendimientos que van desde el 30 - 99\%, dependiendo de las condiciones de reacción (Veljković et al., 2012). Las principales variables que se han asociado al rendimiento son: calidad de material, alcohol utilizado, relación molar alcohol: aceite, temperatura de reacción y parámetros de operación de ultrasonido. Como ejemplo, un estudio previo reportó rendimiento superior de 99,4\% en la transesterificación alcalina del aceite de soya usando una relación molar alcohol: aceite de 6:1, temperatura de $40^{\circ} \mathrm{C}, 15$ minutos de reacción y agitación de ultrasonido (Colucci et al., 2005).

La intención de la presente investigación fue evaluar las condiciones del proceso de elaboración y manufactura del biodiesel usando aceite de corozo (Bactris guineensis) como materia prima e implementando agitación de ultrasonido de sonda directa. Los datos obtenidos fueron ajustados al método de superficie de respuesta para obtener las combinaciones de puntos óptimos que generan el máximo rendimiento.

\section{Metodología}

\section{Materia prima}

Los desechos del fruto de corozo (B. guineensis) fueron colectados en las fruteras cercanas al campus de la Universidad del Atlántico, municipio de Puerto Colombia (1058’59" de latitud norte; 
Producción de biodiesel a partir del aceite extraído de almendra del corozo Bactris guineensis asistida mediante ultrasonido de sonda directa

Madrid De la Rosa., J.M. Mendoza-Meza., D. y Fontalvo-Gómez., M.

Tabla 1. Diseño experimental $2^{3}$ para la extracción por método Soxhlet

\begin{tabular}{|c|c|c|c|}
\hline Tratamientos & $\begin{array}{c}\text { Cantidad de } \\
\text { materia prima (g) }\end{array}$ & $\begin{array}{c}\text { Tiempo de } \\
\text { extracción (h) }\end{array}$ & $\begin{array}{c}\text { Tamaño de } \\
\text { partícula }(\mu)\end{array}$ \\
\hline 1 & 30 & 2 & 500 \\
\hline 2 & 60 & 2 & 500 \\
\hline 3 & 30 & 5 & 500 \\
\hline 4 & 60 & 5 & 500 \\
\hline 5 & 30 & 2 & 1000 \\
\hline 6 & 60 & 2 & 1000 \\
\hline 7 & 30 & 5 & 1000 \\
\hline 8 & 60 & 5 & 1000 \\
\hline
\end{tabular}

Fuente: elaboración propia.

7457'0" de longitud Oeste), departamento del Atlántico. Los frutos fueron despulpados y secados al natural para facilitar la separación manual de la almendra; posteriormente, descascarados y molidos para disminuir el tamaño de partícula mediante un molino de martillos, hasta obtener una harina con tamaño de partícula fino (1000 y 500 micras). Finalmente, la harina fue secada en horno a $100^{\circ} \mathrm{C}$ por dos horas.

\section{Extracción de aceite de corozo}

La extracción se realizó utilizando un extractor Soxhlet de $100 \mathrm{~mL}$. El solvente de extracción fue hexano (pureza de 98,5\%). La tabla 1 ilustra el diseño factorial $2^{k}(\mathrm{k}=3)$ que se aplicó para obtener las mejores condiciones de extracción. Las variables independientes fueron: "cantidad de materia prima (g)", "tiempo de extracción (h)" y "tamaño de partícula (micras, $\mu$ )"; la variable respuesta fue "rendimiento de extracción ( \% p/p)". La extracción se llevó a cabo a temperatura constante $\left(80{ }^{\circ} \mathrm{C}\right)$. La cantidad de solvente en cada extracción fue $120 \mathrm{ml}$. Los análisis se realizaron por duplicado.

\section{Refinación y caracterización}

El aceite crudo obtenido bajo las mejores condiciones de extracción, fue refinado parcialmente con un proceso de desgomado según procedimiento descrito previamente (Hernández et al., 2007) para obtener un aceite libre de fosfátidos hidratables y no hidratables. Para la caracterización del aceite refinado se determinaron las propiedades: densidad, índice de acidez e índice de yodo; de acuerdo a 
las normas técnicas colombianas NTC 336, 218 y 283 (ICONTEC, 2016).

\section{Producción de biodiesel}

La reacción se efectuó en un vaso de precipitados de 100 ml. Se empleó metanol como donador de alquilo e hidróxido de potasio ( $\mathrm{KOH}$; pureza $>85 \%$ ) como catalizador. La reacción se desarrolló usando ultrasonido de sonda directa como medio de agitación. El equipo completo de agitación estaba conformado por un procesador de ultrasonido QSONICA Q500, con una potencia nominal de salida de $500 \mathrm{~W}$ y frecuencia de $20 \mathrm{kHz}$, provisto de un generador, convertidor, cable convertidor y la sonda (13 mm de diámetro). Se usó un diseño central compuesto con 4 factores y 3 bloques para evaluar la incidencia de los parámetros, en el rendimiento de la transesterificación. El diseño generó 27 corridas con e1 punto central por cada bloque (tabla 2). Se evaluó el rendimiento de la reacción como la razón entre la cantidad de EMAG producido y la masa de aceite utilizada inicialmente ( \% $\mathrm{p} / \mathrm{p}$ ). La cantidad de catalizador adicionada fue calculada respecto a la cantidad de aceite usado ( $\%$ $\mathrm{p} / \mathrm{p}$ ). Para todas las reacciones se usó $20 \mathrm{~g}$ de aceite de corozo.

\section{Purificación de biodiesel}

El biodiesel obtenido en cada reacción fue purificado mediante el procedimiento descrito previamente (patente WO2016098025A1) (Argentina Patente $n^{\circ}$ WO2016098025A1, 2016). Finalizada la reacción, se adicionó agua al producto en una proporción de $5 \% \mathrm{p} / \mathrm{v}$ respecto del aceite de partida. Dicha mezcla se mantuvo en agitación a una temperatura de $60{ }^{\circ} \mathrm{C}$ durante 40 minutos. Luego se pasó a decantación a una temperatura de $60^{\circ} \mathrm{C}$, y se separó la fase de biodiesel. Luego, a esta fase se añadió, una solución de ácido cítrico $3 \% \mathrm{p} / \mathrm{p}$ en una proporción de $10 \% \mathrm{p} / \mathrm{v}$ respecto del aceite de partida y se realizó decantación a una temperatura de $60{ }^{\circ} \mathrm{C}$, recuperando la fase que contiene el biodiesel. Posteriormente se añadió agua $(\mathrm{pH}: 7,0)$ en una proporción de $10 \%$ p/v respecto del aceite de partida. Finalmente se secó el producto a una temperatura de entre $80{ }^{\circ} \mathrm{C}$ y $110{ }^{\circ} \mathrm{C}$ durante 45 minutos.

\section{Caracterización del producto obtenido}

\section{Caracterización cualitativa del biodiesel}

Cada una de las reacciones se caracterizó a través de espectroscopia de infrarrojo medio/reflexión total atenuada (MIR/ATR). Se utilizó un espectrofotómetro BRUKER modelo ALPHA, con una resolución $8 \mathrm{~nm}$ y una media correspondiente a 32 scans. El tiempo utilizado para obtener los espectros MIR fue de 48 segundos. Este equipo estaba conectado a una computadora equipada con un software que permite importar los datos de los espectros obtenidos. Se compararon los espectros de los 
Producción de biodiesel a partir del aceite extraído de almendra del corozo Bactris guineensis asistida mediante ultrasonido de sonda directa

Madrid De la Rosa., J.M. Mendoza-Meza., D. y Fontalvo-Gómez., M.

Tabla 2. Diseño experimental central compuesto para la transesterificación

\begin{tabular}{|c|c|c|c|c|c|c|c|c|}
\hline \multirow[t]{2}{*}{ Corridas } & $\begin{array}{l}\text { Relación molar } \\
\text { alcohol: aceite. }\end{array}$ & $\begin{array}{c}\text { Catalizador } \\
(\% \mathrm{p} / \mathrm{p})\end{array}$ & $\begin{array}{l}\text { Amplitud } \\
(\%)\end{array}$ & $\begin{array}{c}\text { Tiempo } \\
\text { (min) }\end{array}$ & $\mathbf{x 1}$ & $x 2$ & $x 3$ & $\mathrm{x} 4$ \\
\hline & \multicolumn{4}{|c|}{ Factores } & \multicolumn{4}{|c|}{$\begin{array}{c}\text { Codificación de las } \\
\text { variables }\end{array}$} \\
\hline 1 & 6,0 & 1,5 & 20,0 & 15,0 & -1 & +1 & -1 & -1 \\
\hline 2 & 6,0 & 0,7 & 30,0 & 15,0 & -1 & -1 & +1 & -1 \\
\hline 3 & 6,0 & 0,7 & 20,0 & 30,0 & -1 & -1 & -1 & +1 \\
\hline 4 & 6,0 & 1,5 & 30,0 & 30,0 & -1 & +1 & +1 & +1 \\
\hline 5 & 7,5 & 1,1 & 25,0 & 22,5 & 0 & 0 & 0 & 0 \\
\hline 6 & 9,0 & 0,7 & 20,0 & 15,0 & +1 & -1 & -1 & -1 \\
\hline 7 & 9,0 & 1,5 & 30,0 & 15,0 & +1 & +1 & +1 & -1 \\
\hline 8 & 9,0 & 1,5 & 20,0 & 30,0 & +1 & +1 & -1 & +1 \\
\hline 9 & 9,0 & 0,7 & 30,0 & 30,0 & +1 & -1 & +1 & +1 \\
\hline 10 & 6,0 & 0,7 & 20,0 & 15,0 & -1 & -1 & -1 & -1 \\
\hline 11 & 6,0 & 1,5 & 30,0 & 15,0 & -1 & +1 & +1 & -1 \\
\hline 12 & 6,0 & 1,5 & 20,0 & 30,0 & -1 & +1 & -1 & +1 \\
\hline 13 & 6,0 & 0,7 & 30,0 & 30,0 & -1 & -1 & +1 & +1 \\
\hline 14 & 7,5 & 1,1 & 25,0 & 22,5 & 0 & 0 & 0 & 0 \\
\hline 15 & 9,0 & 1,5 & 20,0 & 15,0 & +1 & +1 & -1 & -1 \\
\hline 16 & 9,0 & 0,7 & 30,0 & 15,0 & +1 & -1 & +1 & -1 \\
\hline 17 & 9,0 & 0,7 & 20,0 & 30,0 & +1 & -1 & -1 & +1 \\
\hline 18 & 9,0 & 1,5 & 30,0 & 30,0 & +1 & +1 & +1 & +1 \\
\hline 19 & $4,5^{*}$ & 1,1 & 25,0 & 22,5 & -2 & 0 & 0 & 0 \\
\hline 20 & 7,5 & $0,3^{*}$ & 25,0 & 22,5 & 0 & -2 & 0 & 0 \\
\hline 21 & 7,5 & $1,9^{*}$ & 25,0 & 22,5 & 0 & +2 & 0 & 0 \\
\hline 22 & 7,5 & 1,1 & 25,0 & 22,5 & 0 & 0 & 0 & 0 \\
\hline 23 & 7,5 & 1,1 & $15,0^{*}$ & 22,5 & 0 & 0 & -2 & 0 \\
\hline 24 & 7,5 & 1,1 & $35,0^{*}$ & 22,5 & 0 & 0 & +2 & 0 \\
\hline 25 & 7,5 & 1,1 & 25,0 & $7,5^{*}$ & 0 & 0 & 0 & -2 \\
\hline 26 & 7,5 & 1,1 & 25,0 & $37,5^{*}$ & 0 & 0 & 0 & +2 \\
\hline 27 & $10,5^{*}$ & 1,1 & 25,0 & 22,5 & +2 & 0 & 0 & 0 \\
\hline
\end{tabular}

*Puntos axiales definidos por el diseño.

Fuente: elaboración propia. 
Producción de biodiesel a partir del aceite extraído de almendra del corozo Bactris guineensis asistida mediante ultrasonido de sonda directa

Madrid De la Rosa., J.M. Mendoza-Meza., D. y Fontalvo-Gómez., M.

Tabla 3. Normas técnicas colombianas para caracterización de biodiesel

\begin{tabular}{|c|c|c|}
\hline Propiedad & Unidad & Método de ensayo \\
\hline Densidad a $15{ }^{\circ} \mathrm{C}$ & $\mathrm{kg} / \mathrm{m}^{3}$ & ASTM D4052 \\
\hline Número ácido & $\mathrm{mg} \mathrm{KOH} / \mathrm{g}$ & ASTM D664 \\
\hline Contenido en metanol & $\%(\mathrm{~m} / \mathrm{m})$ & ISO 14110 \\
\hline Contenido en monoglicéridos & $\%(\mathrm{~m} / \mathrm{m})$ & ISO 14105 \\
\hline Contenido en diglicéridos & $\%(\mathrm{~m} / \mathrm{m})$ & ISO 14105 \\
\hline Contenido en triglicéridos & $\%(\mathrm{~m} / \mathrm{m})$ & ISO1410 \\
\hline Glicerina libre & $\%(\mathrm{~m} / \mathrm{m})$ & ISO 14105 \\
\hline Glicerina total & $\%(\mathrm{~m} / \mathrm{m})$ & ISO 14105 \\
\hline
\end{tabular}

Fuente: elaboración propia.

productos de las reacciones experimentales, un espectro de una muestra de biodiesel de palma y un espectro de una muestra de aceite de corozo.

\section{Caracterización fisicoquímica del biodiesel}

El biodiesel fue caracterizado según los procedimientos señalados en la norma nacional vigente NTC 5444 y los valores conseguidos fueron comparados con los establecidos en dicha legislación, tal como se detalla en la tabla 3.

\section{Análisis estadístico}

Los datos experimentales obtenidos para cada una de los diseños experimentales se analizaron con el software Statgraphics Centurion (XV) 16.1.15. Se aplicó análisis de varianza (Anova) para estimar el impacto de cada uno de los factores principales y sus interacciones sobre la variable de respuesta estudiada (rendimiento de extracción y rendimiento de reacción), con un nivel de confianza del $95 \%$.

\section{Resultados}

\section{Extracción del aceite}

La tabla 4 presenta los rendimientos de extracción del aceite de corozo con el método Soxhlet, bajo las diferentes condiciones experimentales. 
Producción de biodiesel a partir del aceite extraído de almendra del corozo Bactris guineensis asistida mediante ultrasonido de sonda directa

Madrid De la Rosa., J.M. Mendoza-Meza., D. y Fontalvo-Gómez., M.

Tabla 4. Resultados del diseño experimental $2^{3}$ para la extracción del aceite de corozo

\begin{tabular}{|c|c|c|c|c|}
\hline Tratamiento & $\begin{array}{c}\text { Cantidad de } \\
\text { materia prima (g) }\end{array}$ & $\begin{array}{c}\text { Tiempo de } \\
\text { extracción (h) }\end{array}$ & $\begin{array}{c}\text { Tamaño de } \\
\text { partícula }(\mu)\end{array}$ & $\begin{array}{c}\text { Rendimiento de } \\
\text { extracción ( \% p/p) } \\
\text { (media } \pm \text { DE) }\end{array}$ \\
\hline 1 & 30 & 2 & 500 & $39,43 \pm 0,49$ \\
\hline 2 & 60 & 2 & 500 & $36,94 \pm 0,11$ \\
\hline 3 & 30 & 5 & 500 & $38,04 \pm 0,55$ \\
\hline 4 & 60 & 5 & 500 & $39,27 \pm 0,77$ \\
\hline 5 & 30 & 2 & 1000 & $35,96 \pm 0,48$ \\
\hline 6 & 60 & 2 & 1000 & $34,89 \pm 0,25$ \\
\hline 7 & 30 & 5 & 1000 & $36,06 \pm 0,88$ \\
\hline 8 & 60 & 5 & 1000 & $38,58 \pm 0,16$ \\
\hline
\end{tabular}

*El volumen de solvente gastado durante cada extracción fue de $120 \mathrm{ml}$

Fuente: elaboración propia.

El Anova mostró que el tamaño de partícula de la materia prima (harina de la almendra de corozo) influye significativamente (Anova: $\mathrm{F}=58,77$; valor-p = 0,0001) en la variable respuesta "rendimiento de extracción del aceite", observándose un efecto negativo. Los rendimientos más altos se obtuvieron con el menor tamaño de partícula $(500 \mu$ ), confirmándose que al incrementar la superficie de contacto entre el solvente y el soluto se incrementa la difusión del aceite, lo que favorece la extracción (Farías y Matos, 2009). El tiempo de extracción también contribuyó significativamente en el rendimiento de extracción (Anova: $\mathrm{F}=20,08$; valor-p = 0,002), obteniéndose una relación positiva entre este factor y la variable respuesta, por lo que se deduce que un mayor tiempo de contacto entre el soluto y el solvente lleva a la obtención de una mayor concentración del producto deseado (Cardona, Ríos, y Restrepo, 2006). Las interacciones entre "tamaño de partícula" y "tiempo de extracción" (Anova: F=7,27; valor$\mathrm{p}=0,03$ ) y entre "tamaño de partícula" y "cantidad de materia prima" (Anova: $\mathrm{F}=6,53$; valor-p=0,03) también tuvieron efecto significativo sobre el rendimiento de extracción (Figura 1). Por otra parte, la cantidad de materia prima no tuvo influencia significativa sobre el rendimiento de la extracción del aceite (Anova: $\mathrm{F}=0,04$; valor-p = 0,85), bajo las condiciones experimentales estudiadas; no obstante, la interacción "cantidad de materia prima" y "tiempo de extracción" influyó significativamente en el rendimiento (Anova: $\mathrm{F}=47,42$; valor-p = 0,0001). Por lo tanto, las mejores condiciones fueron: cantidad de materia prima de 30 g, tiempo de extracción 2 h y tamaño de partícula de $500 \mu$. Estas fueron las condiciones usadas para obtener el aceite usado para la transesterificación. 
Producción de biodiesel a partir del aceite extraído de almendra del corozo Bactris guineensis asistida mediante ultrasonido de sonda directa

Madrid De la Rosa., J.M. Mendoza-Meza., D. y Fontalvo-Gómez., M.

Diagrama de Pareto Estandarizada para Rendimiento

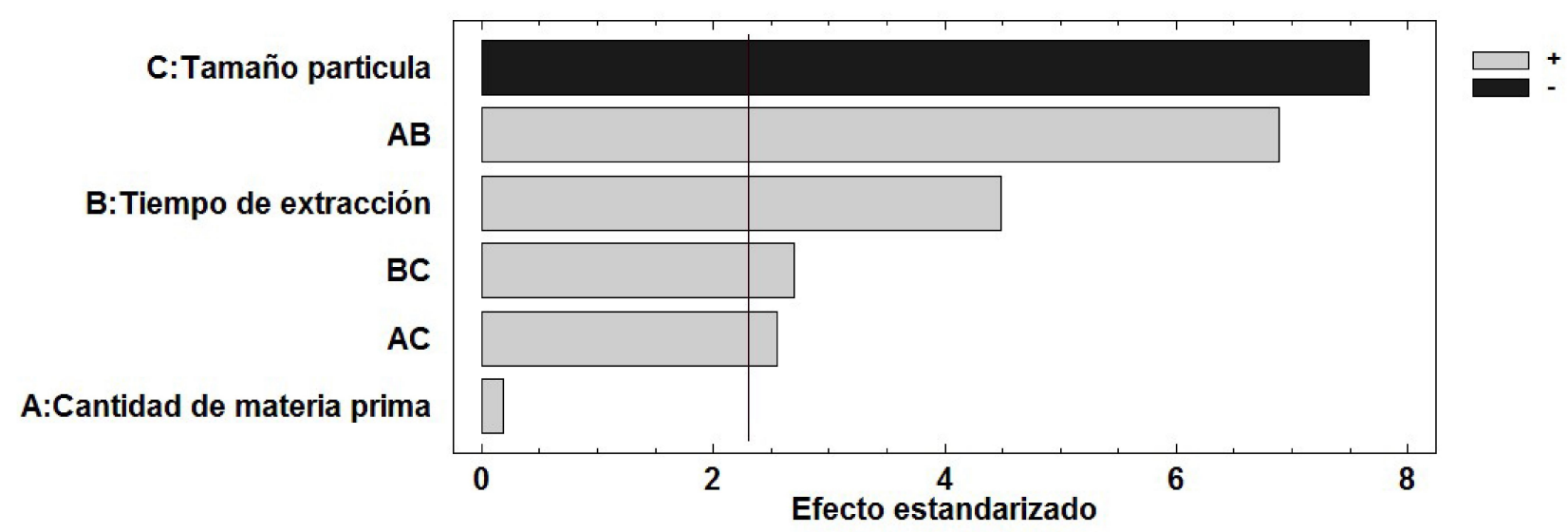

Figura 1. Gráfica de Pareto estandarizado para el diseño experimental $2^{3}$ para la extracción del aceite de corozo Fuente: elaboración propia.

\section{Caracterización del aceite refinado}

La tabla 5 contiene los resultados de la caracterización realizada al aceite refinado de corozo.

Tabla 5. Propiedades físicoquímicas del aceite de corozo

\begin{tabular}{|c|c|c|}
\hline Propiedad & Valor & Unidades \\
\hline Densidad & $0,90 \pm 0,0024$ & $\mathrm{~g} / \mathrm{mL}$ \\
\hline Acidez & $0,36 \pm 0,01$ & $\%$ de ácido láurico \\
\hline Índice de acidez & $1,02 \pm 0,02$ & $\mathrm{mg} \mathrm{KOH} / \mathrm{g}$ de muestra \\
\hline Índice de yodo & $4,47 \pm 0,45$ & $\mathrm{~g}$ de $\mathrm{I}_{2} / 100 \mathrm{~g}$ de muestra \\
\hline
\end{tabular}

Fuente: elaboración propia.

La densidad, índice de acidez e índice de yodo son parámetros a considerarse en la caracterización del aceite destinado a la obtención de biodiesel (Tacias et al., 2016). La densidad del aceite de corozo a $25^{\circ} \mathrm{C}(0,901 \mathrm{~g} / \mathrm{ml})$ fue consistente a la reportada previamente por (Polo et al., 2018); además, fue similar a la descrita para los aceites de palma africana $\left(0,925 \mathrm{~g} / \mathrm{ml}\right.$ a $\left.25^{\circ} \mathrm{C}\right)$ (Albis et al., 2005), palma de coco $(0,917 \mathrm{~g} / \mathrm{ml})$, aceite de colza $\left(0,905 \mathrm{~g} / \mathrm{ml}\right.$ a $\left.25^{\circ} \mathrm{C}\right)$ y aceite de soya $(0,916 \mathrm{~g} / \mathrm{ml})$ (Sahasrabudhe et al., 2017).

El índice de acidez fue de 0,36\% (expresado en porcentaje de ácido láurico). Este parámetro de- 
Producción de biodiesel a partir del aceite extraído de almendra del corozo Bactris guineensis asistida mediante ultrasonido de sonda directa

Madrid De la Rosa., J.M. Mendoza-Meza., D. y Fontalvo-Gómez., M.

termina la cuantía de ácidos grasos libres que contiene el aceite (Rivera et al., 2014) y define el proceso que se debe seguir para la transesterificación. Aceites con valores de índice de acidez superior al $1 \%$ requieren un tratamiento previo de esterificación de ácidos grasos libres, para evitar formación de jabones (Phan y Phan, 2008). Los jabones y otros sub-productos en el biodiesel dificultan las técnicas de recuperación y purificación del biocombustible, por tal razón su formación debe evitarse (Tacias et al., 2016).

El índice de yodo, es la medida de insaturaciones en el aceite (González et al., 2012). Según (Toscano y Maldini, 2007) los aceites destinados a la producción de biodiesel deberán tener valores de índice de yodo menores o iguales a $120 \mathrm{~g}$ de $\mathrm{I}_{2} / 100 \mathrm{~g}$ de muestra. El aceite de corozo obtenido en el presente estudio mostró un valor de índice de yodo de $4,47 \mathrm{~g} \mathrm{de} \mathrm{I}_{2} / 100 \mathrm{~g}$ de muestra, lo que indica un bajo contenido de ácidos grasos insaturados, haciéndolo menos susceptible a la oxidación (Benjumea et al., 2009). Por otra parte, el nivel de insaturación del biodiesel tiene una relación inversa con el índice de cetano; es decir, a menor índice de yodo mayor índice de cetano y en consecuencia una mejor calidad de ignición del biocombustible en los motores diésel (Lamaisri et al., 2015). Por tanto, un aceite con índice de yodo bajo será más conveniente para la producción biodiesel.

\section{Producción de biodiesel}

La tabla 6 muestra los resultados experimentales obtenidos con el diseño central compuesto para establecer las mejores condiciones de reacción en la transesterificación asistida por ultrasonido del aceite de almendra de corozo.

El Anova mostró un efecto significativo de la variable "concentración de catalizador" en el rendimiento de reacción (Anova: $\mathrm{F}=7,96$; valor-p = 0,02); también manifestó un efecto cuadrático significativo de la variable "Tiempo de reacción" (Anova: $F=10,31$; valor-p $=0,01$ ) La otras variables estudiadas, "relación molar alcohol: aceite" (Anova: F =3,23; valor-p: 0,10) y "amplitud del ultrasonido" (Anova: $\mathrm{F}=2,05$; valor-p =0,18), no influyeron significativamente en el rendimiento, con un intervalo de confianza del $95 \%$, ilustrado en la Figura 2.

El efecto de la concentración de catalizador sobre el rendimiento de la reacción de transesterificación se ha descrito previamente (Atadashi et al., 2013) estableciendo que elevadas concentraciones de $\mathrm{KOH}$ disminuyen el rendimiento de los metilésteres y propicia la aparición de jabones a causa de la saponificación de los ácidos grasos en presencia de agua (Ruiz et al., 2016). El gráfico de Pareto (Figura 2) indica un efecto negativo de la concentración de $\mathrm{KOH}$ sobre el rendimiento de reacción, lo cual está en proporción a lo reportado en la literatura para diferentes aceites vegetales. Con el aceite de corozo el porcentaje de conversión más alto (81,05\%) se obtuvo con 0,70\% de $\mathrm{KOH}$, mientras que el más bajo $(67,41 \%)$ se obtuvo con 1,1 \% de $\mathrm{KOH}$. Este resultado fue similar al reportado por (Stavarache et al., 2003) quienes obtuvieron rendimientos entre $75-98 \%$ con $0,50 \%$ en peso de $\mathrm{NaOH}$ como catalizador y una relación alcohol: aceite de 6:1, usando agitación con sonda de ultrasonido a $40 \mathrm{kHz}$ de frecuencia. 
Producción de biodiesel a partir del aceite extraído de almendra del corozo Bactris guineensis asistida mediante ultrasonido de sonda directa

Madrid De la Rosa., J.M. Mendoza-Meza., D. y Fontalvo-Gómez., M.

Tabla 6. Resultados del diseño central compuesto para la transesterificación

\begin{tabular}{|c|c|c|c|c|c|}
\hline Corrida & $\begin{array}{l}\text { Relación molar } \\
\text { alcohol: aceite. }\end{array}$ & $\begin{array}{c}\text { Catalizador } \\
(\% \mathrm{p} / \mathrm{p})\end{array}$ & $\begin{array}{l}\text { Amplitud } \\
\text { (\%) }\end{array}$ & $\begin{array}{c}\text { Tiempo } \\
\text { (min) }\end{array}$ & $\begin{array}{l}\text { Rendimiento de } \\
\text { reacción }(\% \mathrm{p} / \mathrm{p})\end{array}$ \\
\hline 1 & 6,0 & 1,5 & 20,0 & 15,0 & 80,58 \\
\hline 2 & 6,0 & 0,7 & 30,0 & 15,0 & 78,84 \\
\hline 3 & 6,0 & 0,7 & 20,0 & 30,0 & 78,09 \\
\hline 4 & 6,0 & 1,5 & 30,0 & 30,0 & 70,95 \\
\hline 5 & 7,5 & 1,1 & 25,0 & 22,5 & 78,34 \\
\hline 6 & 9,0 & 0,7 & 20,0 & 15,0 & 79,95 \\
\hline 7 & 9,0 & 1,5 & 30,0 & 15,0 & 70,89 \\
\hline 8 & 9,0 & 1,5 & 20,0 & 30,0 & 76,18 \\
\hline 9 & 9,0 & 0,7 & 30,0 & 30,0 & 78,48 \\
\hline 10 & 6,0 & 0,7 & 20,0 & 15,0 & 81,05 \\
\hline 11 & 6,0 & 1,5 & 30,0 & 15,0 & 77,02 \\
\hline 12 & 6,0 & 1,5 & 20,0 & 30,0 & 77,46 \\
\hline 13 & 6,0 & 0,7 & 30,0 & 30,0 & 77,94 \\
\hline 14 & 7,5 & 1,1 & 25,0 & 22,5 & 77,45 \\
\hline 15 & 9,0 & 1,5 & 20,0 & 15,0 & 73,82 \\
\hline 16 & 9,0 & 0,7 & 30,0 & 15,0 & 80,34 \\
\hline 17 & 9,0 & 0,7 & 20,0 & 30,0 & 79,03 \\
\hline 18 & 9,0 & 1,5 & 30,0 & 30,0 & 73,24 \\
\hline 19 & 4,5 & 1,1 & 25,0 & 22,5 & 79,27 \\
\hline 20 & 7,5 & 0,3 & 25,0 & 22,5 & 75,91 \\
\hline 21 & 7,5 & 1,9 & 25,0 & 22,5 & 75,76 \\
\hline 22 & 7,5 & 1,1 & 25,0 & 22,5 & 78,48 \\
\hline 23 & 7,5 & 1,1 & 15,0 & 22,5 & 71,67 \\
\hline 24 & 7,5 & 1,1 & 35,0 & 22,5 & 72,30 \\
\hline 25 & 7,5 & 1,1 & 25,0 & 7,5 & 69,94 \\
\hline 26 & 7,5 & 1,1 & 25,0 & 37,5 & 67,41 \\
\hline 27 & 10,5 & 1,1 & 25,0 & 22,5 & 73,48 \\
\hline
\end{tabular}

Fuente: elaboración propia. 
Diagrama de Pareto Estandarizada para Rendimiento

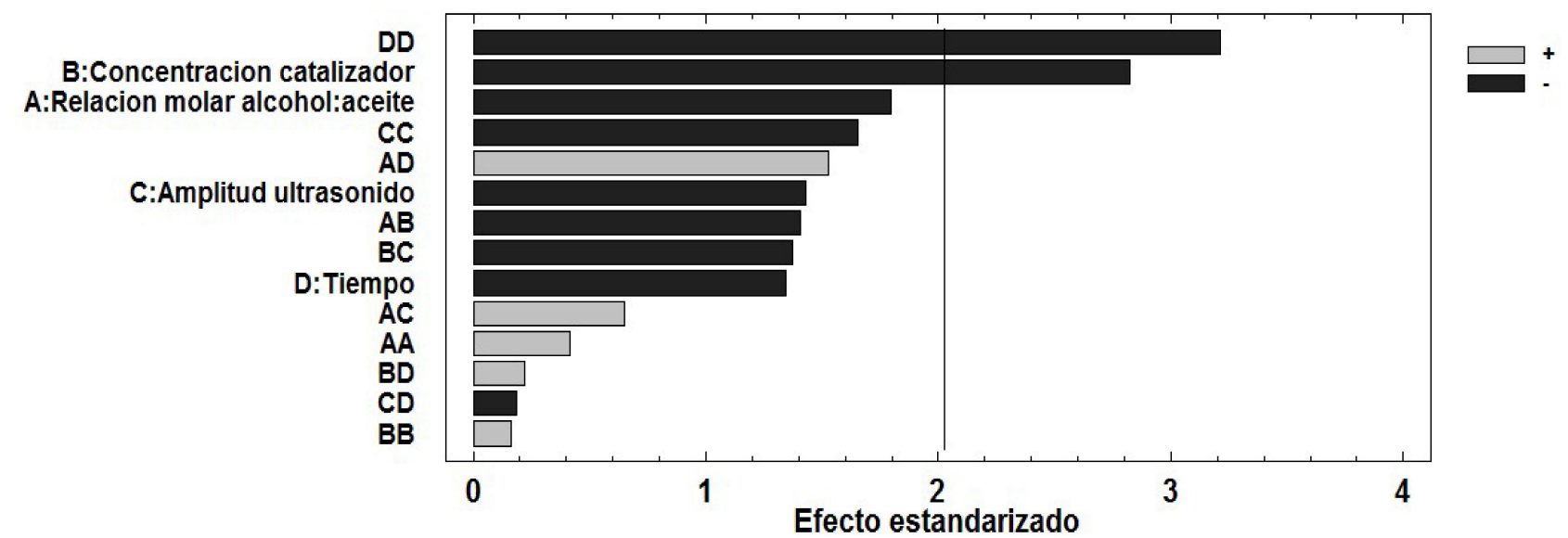

Figura 2. Gráfica de Pareto estandarizado para el diseño central compuesto para la transesterificación de aceite

Fuente: elaboración propia.

En cuanto al tiempo, el gráfico de Pareto (Figura 2) mostró un efecto negativo de esta variable sobre la reacción, obteniéndose el rendimiento más alto en 15 minutos de reacción. Estudios previos revelan que en general, la formación de FAMEs es considerable en los primeros minutos de reacción (con agitación constante y condiciones óptimas), pero es aproximadamente inexistente después de 90 minutos (Torres et al., 2017), así lo confirman (Fontalvo et al., 2013), quienes evaluaron el desarrollo de la transesterificación alcalina del aceite de canola mediante espectroscopía de infrarrojo cercano (NIR) y Raman de forma simultánea, encontrando que los cambios espectrales más significativos ocurren durante los primeros 40 minutos de reacción, después de este tiempo, los cambios son menos marcados. (Topare et al., 2019), reportaron rendimientos superiores al $80 \%$ entre 15 - 40 minutos de la transesterificación asistida por ultrasonido de aceite de soya usando $\mathrm{KOH}$ como catalizador. (Encinar et al., 2018) obtuvieron rendimientos de reacción del $95 \%$ en la transesterificación del aceite de colza usando una potencia ultrasónica de $320 \mathrm{~W}$, concentración de catalizador $\mathrm{KOH}$ del 0,7\%, y una relación molar alcohol: aceite 9:1. Estos comportamientos son consistentes con los obtenidos en el presente estudio.

Aunque bajo las condiciones experimentales del presente estudio, el modelo estadístico mostró un efecto no significativo de la variable "amplitud del ultrasonido", en la gráfica de superficie de respuesta se observa que a mayor amplitud los rendimientos de reacción disminuyen (Figura 3). Estudios previos han demostrado que el mezclado por ultrasonido puede incidir positivamente en el proceso de transesterificación. Las ondas ultrasónicas generan cavitación, que consiste en el surgimiento, aumento y ruptura implosiva de burbujas dentro del líquido irradiado (Veljković et al., 
Producción de biodiesel a partir del aceite extraído de almendra del corozo Bactris guineensis asistida mediante ultrasonido de sonda directa

Madrid De la Rosa., J.M. Mendoza-Meza., D. y Fontalvo-Gómez., M.

Nota: las variables relación molar alcohol-aceite y el tiempo de reacción se fijaron en los valores

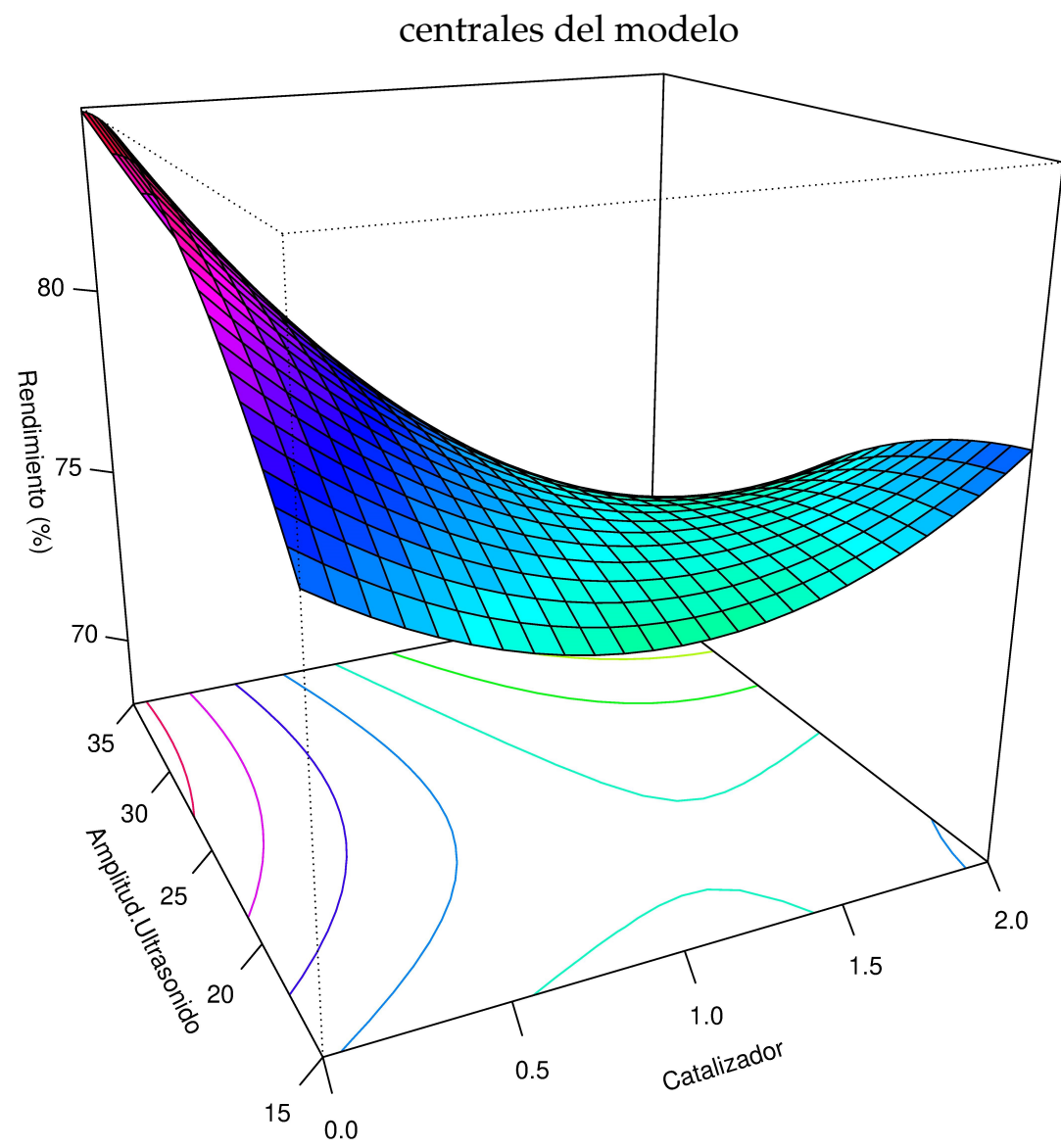

Figura 3. Superficie de respuesta estimada para el rendimiento de reacción de transesterificación del aceite de corozo (Bactris guineensis)

Fuente: elaboración propia.

2012, Colucci et al., 2005). Las burbujas de cavitación dan lugar a la micro-mezcla entre las fases del aceite y metanol, lo que incrementa la transferencia de masa y calor entre reactantes favoreciendo la formación de FAME. No obstante, valores altos de amplitud ultrasónica o potencias ultrasónicas aplicadas por largos intervalos de tiempo pueden destruir el efecto de cavitación (Sun et al., 2018), ocasionando pérdida de la homogeneización de los componentes iniciales y, en consecuencia, una disminución del rendimiento final de la transesterificación.

La relación molar alcohol: aceite es otro de los factores que hay que ajustar en la reacción. La estequiometría de la transesterificación indica que esta relación debe ser 3 moles de metanol por cada mol de aceite (3:1). Sin embargo, al ser la transesterificación una reacción reversible, se requiere de una relación alcohol: aceite mayor para potenciar la formación de productos (Lee y Saka, 2010), aumentar la solubilidad entre reactantes y favorecer el choque efectivo entre las fases de triglicérido y el 
alcohol (Musa, 2016). En el presente estudio, el mayor rendimiento experimental ( $81,05 \%)$ se obtuvo usando una relación molar alcohol: aceite de 6:1 y 15 minutos de reacción asistida por ultrasonido. Este resultado es comparable al obtenido por (Stavarache et al., 2006), y (Fan et al., 2010), quienes lograron obtener conversiones de biodiesel del 98 \% usando una relación molar alcohol: aceite de 6:1, en 20 y 8 min de irradiación de ultrasonido, respectivamente.

\section{Caracterización cualitativa del biodiesel}

El biodiesel de corozo obtenido bajo las mejores condiciones de reacción se caracterizó inicialmente por espectroscopía MIR/ATR. La figura 4 presenta una comparación de los espectros del aceite de corozo, biodiesel de corozo y biodiesel de palma; este último utilizado como referencia para el biodiesel de corozo.

La tabla 7 muestra las regiones del espectro MIR que caracterizan al aceite de corozo y sus metilésteres. Se destaca una señal a $1436 \mathrm{~cm}^{-1}$, propia de la deformación asimétrica del grupo $\mathrm{O}=\mathrm{C}-\mathrm{O}-\mathrm{CH}_{3}$, la cual es característica del biodiesel (Andrade et al., 2016) y está ausente en el espectro de aceite, lo que confirma la conversión de los acilglicéridos en FAMEs. Por otra parte, la señal de $1378 \mathrm{~cm}^{-1}$ correspondiente a las vibraciones de flexión de los grupos $\mathrm{CH}_{2}$ y $\mathrm{CH}_{3}$ (Jović, 2016) se encontró únicamente en el aceite de corozo.

\section{Caracterización fisicoquímica del biodiesel}

Se evaluaron algunas propiedades de calidad del biodiesel (Ver Tabla 8). Los análisis fueron realizados de acuerdo a protocolos establecidos en la empresa Biocombustibles Sostenibles del Caribe (BIOSC) y se interpretaron de acuerdo a lo establecido en la norma NTC 5444 del Instituto Colombiano de Normas Técnicas y Certificación.

Densidad: El valor de la densidad del biodiesel de corozo está dentro de lo establecido por la norma. Valores por fuera del estándar podrían generar dificultades en el proceso de combustión (Arias et al., 2011) ya que la densidad es una propiedad que influye en la cantidad de masa de combustible que es inyectada al motor (Pratas et al., 2011).

Número ácido: Un combustible con un alto valor de número ácido tiende a corroer el tanque de combustible y las tuberías del motor, además, dicha acidez aumenta con el tiempo de almacenamiento (Kakati y Gogoi, 2016). El valor de ácido del biodiesel de corozo (Bactris guineensis) cumple con el límite establecido por la norma NTC 5444 y es bajo comparado con lo obtenido de especies como Jatropha Curcas (0,40 mg KOH/g) (Kumar et al., 2007) y Karanja (0,23 mg KOH/g) (Sahoo y Das, 2009).

Contenido de monoglicéridos, diglicéridos y triglicéridos: El valor del contenido de monoglicéridos fue de $0,18 \% \mathrm{~m} / \mathrm{m}$; menor al límite máximo recomendado por la norma $(0,80 \% \mathrm{~m} / \mathrm{m})$. Por otro lado, tanto el contenido de diglicéridos como el contenido de triglicéridos tuvieron ambos un valor de 

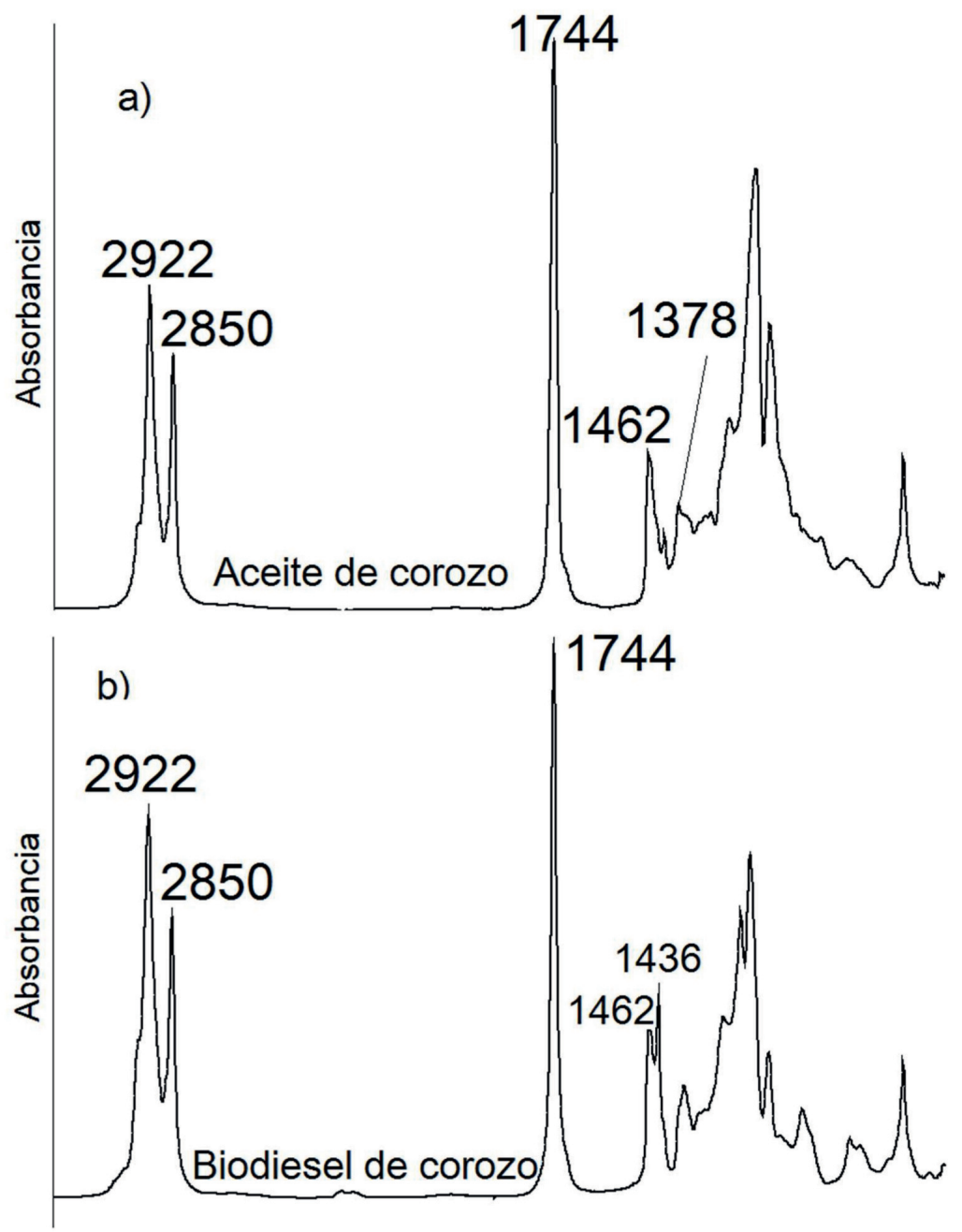

1744

c)
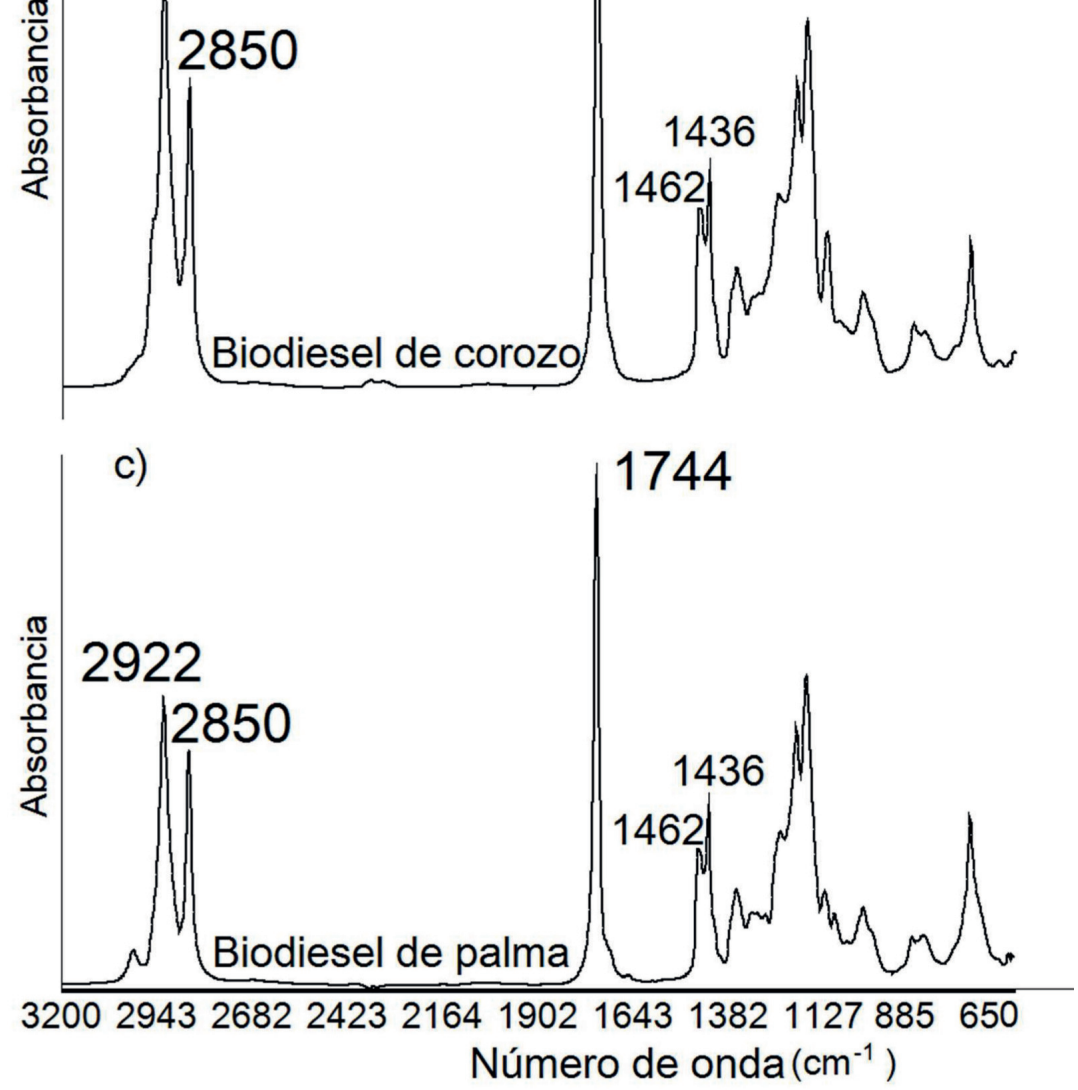

Figura 4. Comparación de los espectros MIR del a) aceite de corozo, b) biodiésel del corozo y c) biodiésel de palma

Fuente: elaboración propia. 
Producción de biodiesel a partir del aceite extraído de almendra del corozo Bactris guineensis asistida mediante ultrasonido de sonda directa

Madrid De la Rosa., J.M. Mendoza-Meza., D. y Fontalvo-Gómez., M.

Tabla 7. Regiones características del espectro de aceite y biodiesel de corozo

\begin{tabular}{|c|c|c|c|c|c|}
\hline \multirow{2}{*}{ Región } & \multirow{2}{*}{$\begin{array}{c}\text { Rango de } \\
\text { número de } \\
\text { onda }\left(\mathrm{cm}^{-1}\right)\end{array}$} & \multirow{2}{*}{ Tipo de vibración } & \multicolumn{3}{|c|}{ Absorción $\left(\mathrm{cm}^{-1}\right)$} \\
\hline & & & $\begin{array}{c}\text { Aceite de } \\
\text { corozo }\end{array}$ & $\begin{array}{l}\text { Biodiésel de } \\
\text { corozo }\end{array}$ & $\begin{array}{c}\text { Biodiésel de } \\
\text { palma }\end{array}$ \\
\hline 1 & 2809-3012 & $\begin{array}{l}\text { vas }\left(\mathrm{CH}_{2}\right) \text { y vs } \\
\text { correspondiente a } \\
\text { grupos metileno }\end{array}$ & $2922-2850$ & $2922-2850$ & $2922-2850$ \\
\hline 2 & $1763-1712$ & $\begin{array}{c}\mathrm{v}(>\mathrm{C}=\mathrm{O}) \\
\text { correspondiente } \\
\text { a ésteres }\end{array}$ & 1744 & 1744 & 1744 \\
\hline \multirow[t]{2}{*}{3} & \multirow[t]{2}{*}{$1425-1477$} & $\begin{array}{c}\text { vas }\left(\mathrm{O}-\mathrm{CH}_{3}\right) \\
\text { correspondiente a } \\
\text { los metilésteres }\end{array}$ & 1436 & No detectado & No detectado \\
\hline & & $\begin{array}{c}\delta \mathrm{as}\left(\mathrm{CH}_{2}\right) / \delta \mathrm{as}\left(\mathrm{CH}_{3}\right) \\
\text { correspondiente a los } \\
\text { grupos metileno }\end{array}$ & 1462 & 1462 & 1462 \\
\hline 4 & $1370-1400$ & $\begin{array}{c}\text { vas }\left(\mathrm{O}-\mathrm{CH}_{2}\right) \\
\text { correspondiente } \\
\text { de glicerol, } \\
\text { triacilglicéridos, } \\
\text { diacilglicéridos y } \\
\text { monoacilglicéridos }\end{array}$ & 1378 & No detectado & No detectado \\
\hline 5 & $1357-1423$ & $\begin{array}{l}\text { vs }(\mathrm{C}-\mathrm{O}) \text { asignado } \\
\mathrm{COO}^{-} \text {grupos de } \\
\text { ácidos carboxílicos }\end{array}$ & 1415 & 1360 & 1362 \\
\hline
\end{tabular}

\section{Notas:}

a. $\mathrm{v}=$ Extensión, $\delta=$ flexión, as= asimétrica, $\mathrm{s}=$ simétrica.

b. Los rangos de longitud de onda $\left(\mathrm{cm}^{-1}\right)$ se basan en datos reportados en estudios similares

Fuente: (Dilek et al., 2012, Mahamuni y Adewuyi, 2009).

$0,03 \% \mathrm{~m} / \mathrm{m}$ e igualmente cumplen con los requerimientos exigidos por la normativa vigente. 
Producción de biodiesel a partir del aceite extraído de almendra del corozo Bactris guineensis asistida mediante ultrasonido de sonda directa

Madrid De la Rosa., J.M. Mendoza-Meza., D. y Fontalvo-Gómez., M.

Tabla 8. Valores de las propiedades fisicoquímicas del biodiésel de corozo comparados con la norma nacional vigente

\begin{tabular}{|c|c|c|c|}
\hline Propiedad & Unidad & Valor & Valor norma \\
\hline Densidad a $15^{\circ} \mathrm{C}$ & $\mathrm{kg} / \mathrm{m}^{3}$ & 870 & $860-900$ \\
\hline Número ácido & $\mathrm{mg} \mathrm{KOH} / \mathrm{g}$ & 0,12 & 0,50 \\
\hline Contenido en metanol & $\%(\mathrm{~m} / \mathrm{m})$ & 0,04 & 0,20 \\
\hline Contenido en monoglicéridos & $\%(\mathrm{~m} / \mathrm{m})$ & 0,18 & 0,80 \\
\hline Contenido en diglicéridos & $\%(\mathrm{~m} / \mathrm{m})$ & 0,03 & 0,20 \\
\hline Contenido en triglicéridos & $\%(\mathrm{~m} / \mathrm{m})$ & 0,03 & 0,20 \\
\hline Glicerina libre & $\%(\mathrm{~m} / \mathrm{m})$ & 0,45 & 0,02 \\
\hline Glicerina total & $\%(\mathrm{~m} / \mathrm{m})$ & 0,51 & 0,25 \\
\hline
\end{tabular}

Fuente: NTC 5444 ( (ICONTEC, 2016), NTC 5444, Biodiesel para uso en motores diesel, 2006).

\section{Conclusiones}

En este estudio, se optimizaron las condiciones de extracción del aceite de la almendra de corozo (Bactris guineensis) a partir de la biomasa residual del fruto, demostrando el valor agregado que se le puede dar a este recurso natural. El aceite de corozo fue apto para la producción de FAME a través de catálisis básica usando agitación con ultrasonido por sonda directa, obteniéndose un rendimiento de reacción máximo de $81,05 \%$ p/p. A través de un diseño experimental de superficie de respuesta central compuesto se determinó que, bajo las condiciones experimentales usadas, la concentración de catalizador y el tiempo de reacción son los factores con mayor repercusión en el rendimiento de la reacción de transesterificación. La agitación con sonda de ultrasonido permitió reducir los gastos energéticos asociados con la producción del biocombustible (rendimientos superiores de $70 \%$ en 15 minutos). Las propiedades del producto final cumplieron los procedimientos establecidos en la norma técnica colombiana 5444.

\section{Financiamiento}

Este trabajo fue financiado con recursos del proyecto "Desarrollo de metodologías ecoamigables para el aprovechamiento de residuos generados en diversas industrias de la Región del Caribe colombiano, de la séptima convocatoria interna para el fortalecimiento de los grupos de investigación" y con el apoyo del programa Jóvenes Investigadores de Colciencias, Convocatoria "N 775 de 2017". 


\section{Agradecimientos}

Los autores agradecen a la empresa Biocombustibles Sostenibles del Caribe (BIOSC) por el apoyo técnico en los análisis fisicoquímicos de calidad del biodiesel de corozo.

\section{Referencias}

[ICONTEC, 2016] (ICONTEC), I. C. (2006). NTC 5444, Biodiesel para uso en motores diesel. Bogotá: ICONTEC. $\uparrow$ Ver página 68

[ICONTEC, 2016] (ICONTEC), I. C. (2016). NTC 254. Bogotá: ICONTEC. Obtenido de https : // tienda.icontec.org/wp-content/uploads/pdfs/NTC254.pdf 个Ver página 56

[Albis et al., 2005] Albis, A., Parra, J., y Sánchez, F. (2005). Transesterificación del aceite de palma con metanol por catálisis heterogénea. Ingeniería e Investigación, 25 (2), 71-77. Obtenido de http://www.scielo.org.co/scielo.php?pid=S0120-56092005000200009\& script=sci_abstract\&tlng=es $\uparrow$ Ver página 60

[Amaringo y Hormaza, 2018] Amaringo, F., y Hormaza, A. (2018). Adsorción de rojo 40 sobre cascarilla de arroz: determinación del equilibrio, cinética y termodinámica. Tecnura, 22(56), 13-28. doi:

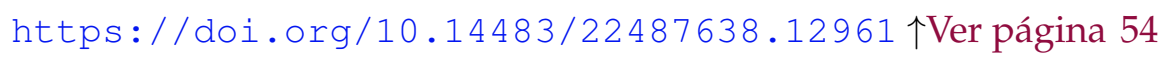

[Andrade et al., 2016] Andrade, M., Parussulo, A., Netto, C., Andrade, L., y Toma, H. (2016). Lipase immobilized on polydopamine-coated magnetite nanoparticles for biodiesel production from soybean oil. Biofuel Research Journal, 3(2), 403-409. doi: https : / doi . org/10 . 18331 /BRJ2016.3. $2.5 \uparrow$ Ver página 53,65

[Arias et al., 2011] Arias, D., E., Teuta, C., y Parra, J. (2011). Caracterización de las propiedades del biodiesel de girasol bajo la Norma NTC de 100/04 y medición de poder calorífico. Revista Avances Investigación en Ingeniería, 8(2), 73-80. Obtenido de https://revistas.unilibre.edu.co/ index.php/avances/article/view/2709/2129个Ver página 65

[Atadashi et al., 2013] Atadashi, I., Aroua, M., Abdul, A., y Sulaiman, N. (2013). The effects of catalysts in biodiesel production: A review. Journal of Industrial and Engineering Chemistry, 19 (1), 14-26. doi: https://doi.org/10.1016/j.jiec.2012.07.009个Ver página 61

[Benjumea et al., 2009] Benjumea, P., Agudelo, J., y Ríos, L. (2009). Biodiésel: Producción, calidad y caracterización. Medellín: Editorial Universidad de Antioquia. $\uparrow$ Ver página 61

[Bernal y Galeano, 2013] Bernal, R., y Galeano, G. (2013). Cosechar sin destruir - Aprovechamiento sostenible de palmas colombianas. Bogotá: Facultad de Ciencias-Instituto de Ciencias Naturales, Universidad Nacional de Colombia. $\uparrow$ Ver página 54 
Producción de biodiesel a partir del aceite extraído de almendra del corozo Bactris guineensis asistida mediante ultrasonido de sonda directa

Madrid De la Rosa., J.M. Mendoza-Meza., D. y Fontalvo-Gómez., M.

[Cardona, Ríos, y Restrepo, 2006] Cardona, E., Ríos, L., y Restrepo, G. (2006). Extracción del carotenoide licopeno del tomate chonto (Lycopersicum esculentum). Vitae, 13(2), 44-53. Obtenido de http://www.scielo.org.co/scielo.php?script=sci_abstract\&pid= S0121-40042006000200006 个Ver página 59

[Colucci et al., 2005] Colucci, J., Borrero, E., y Alape, F. (2005). Biodiesel from an alkaline transesterification reaction of soybean oil using ultrasonic mixing. Journal of the American Oil Chemists' Society, 82(7), 525-530. doi https://doi.org/10.1007/s11746-005-1104-3个Ver página 54, 63

[Dilek et al., 2012] Dilek, D., Udoh, A., Ozer, T., Akbulut, A., Erkaya, I., Yildiz, K., y Guler, D. (2012). Fourier transform infrared (FTIR) spectroscopy for identification of Chlorella vulgaris Beijerinck 1890 and Scenedesmus obliquus (Turpin) Kützing 1833. African Journal of Biotechnology, 11(16), 3817-3824. doi: https://doi.org/10.5897/AJB11.1863 个Ver página 67

[Durán et al., 2018] Durán, D., Figueroa, Á., Gualdrón, M. A., y Sierra, R. (2018). Potential of tropical fruit waste in bioenergy processes and bioproducts design. 26th European Biomass Conference and Exhibition (págs. 166-174). Copenhagen: ResearchGate. doi:10.5071/26thEUBCE2018-1AV.2.18 $\uparrow$ Ver página 53

[Durišić-Mladenović et al., 2018] Durišić-Mladenović, N., Kiss, F., Škrbić, B., Tomić, M., Mićić, R., y Predojević, Z. (2018). Current state of the biodiesel production and the indigenous feedstock potential in Serbia. Renewable and Sustainable Energy Reviews, 81(1), 280-291. doi: https: //doi.org/10.1016/j.rser.2017.07.059个Ver página 54

[Encinar et al., 2018] Encinar, J., Pardal, A., Sánchez, N., y Nogales, S. (2018). Biodiesel by Transesterification of Rapeseed Oil Using Ultrasound: A Kinetic Study of Base-Catalysed Reactions. Energies, 11(1), 2229-2242. doi: https://doi.org/10.3390/en11092229 个Ver página 63

[Esquinas et al., 2018] Esquinas, N., Márquez, G., Permanyer, A., y Gallego, J. (2018). Geochemical evaluation of crude oils from the caracara and tiple areas, eastern llanos basin, Colombia: palaeo biodegradation and oil mixing. Journal of Petroleum Geology, 41(2), 113-134. doi: https: / / doi . org/10.1111/jpg.12696 ^er página 53

[Esteves et al., 2018] Esteves, E., Esteves, V., Bungenstab, D., Araújo, O., y Morgado, C. (2018). Greenhouse gas emissions related to biodiesel from traditional soybean farming compared to integrated crop-livestock systems. Journal of Cleaner Production, 179(1), 81-92. doi: https : / doi . org/10 . $1016 / j \cdot j c l e p r o .2017 .12 .262 \uparrow$ Ver página 53

[Fan et al., 2010] Fan, X., Chen, F., y Wang, X. (2010). Ultrasound-assisted synthesis of biodiesel from crude cottonseed oil using response surface methodology. Journal of Oleo Science, 59(5), 235-241. doi: https://doi.org/10.5650/jos.59.235 个Ver página 65 
Producción de biodiesel a partir del aceite extraído de almendra del corozo Bactris guineensis asistida mediante ultrasonido de sonda directa

Madrid De la Rosa., J.M. Mendoza-Meza., D. y Fontalvo-Gómez., M.

[Farías y Matos, 2009] Farías, A., y Matos, R. (2009). Influencia de la temperatura y tamaño de partícula en el proceso de extracción de aceite de semilla de uva (Vitis vinífera). Revista de investigación universitaria, 1(1), 31-37. Obtenido de https://revistas.upeu.edu.pe/index.php/riu/ article/view/686 个Ver página 59

[Flórez et al., 2016] Flórez, D., Barco, J., y Rincón, S. (2016). Análisis comparativo de la carbonización de cuesco de palma de aceite en reactores de lecho fijo. Tecnura ,20(49), 45-58. doi: https : / / doi . org/10.14483/udistrital.jour.tecnura.2016.3.a03个Ver página 53

[Fontalvo et al., 2013] Fontalvo, M., Colucci, J., Velez, N., y Romañach, R. (2013). In-Line NearInfrared (NIR) and Raman Spectroscopy Coupled with Principal Component Analysis (PCA) for in Situ Evaluation of the Transesterification Reaction. Applied Spectroscopy, 67(10), 1142-1149. doi: https://doi.org/10.1366/12-06729个Ver página 63

[Gaurav et al., 2017] Gaurav, N., Sivasankari, S., Kiran, G., Ninawe, A., y Selvin, J. (2017). Utilization of bioresources for sustainable biofuels: A review. Renewable and sustainable energy reviews, 73(1), 205-214. doi: https://doi.org/10.1016/j.rser.2017.01.070 个Ver página 53

[González et al., 2012] González, D., Benavides, Y., Londoño, J., Restrepo, M., y Cardona, B. (2012). Comparación del aceite de aguacate variedad Hass cultivado en Colombia, obtenido por fluidos supercríticos y métodos convencionales: una perspectiva desde la calidad. Revista Lasallista de investigación, 9(2), 151-161. Obtenido de http://www.scielo.org.co/scielo.php?script= sci_abstract\&pid=S1794-44492012000200016 $\uparrow$ Ver página 61

[Granados y Valencia, 2018] Granados, W., y Valencia, J. (2018). Cadena de palma de aceite Marzo 2018. Ministerio de Agricultura. Bogotá: Ministerio de Agricultura. Obtenido de https://www . scribd.com/document/399412396/002-Cifras-Sectoriales-2018-Marzo-Palma 个Ver página 53

[Hernández et al., 2007] Hernández, C., Mieres, A., Niño, Z., y Pérez, S. (2007). Efecto de la Refinación Física Sobre el Aceite de la Almendra del Corozo (Acrocomia aculeata). Información tecnológica, 18(5), 59-68. doi: https://doi.org/10.4067/S0718-07642007000500008 个Ver página 55

[Jović, 2016] Jović, O. (2016). Durbin-Watson partial least-squares regression applied to MIR data on adulteration with edible oils of different origins. Food Chemistry, 213(1), 791-798. doi: https: //doi.org/10.1016/j.foodchem.2016.07.016个Ver página 65

[Kakati y Gogoi, 2016] Kakati, J., y Gogoi, T. (2016). Biodiesel production from Kutkura (Meyna spinosa Roxb. Ex.) Fruit seed oil: Its characterization and engine performance evaluation with $10 \%$ and $20 \%$ blends. Energy Conversion and Management, 121(1), 152-161. doi: https: //doi.org/ $10.1016 / j$.enconman.2016.05.019 ^Ver página 65 
Producción de biodiesel a partir del aceite extraído de almendra del corozo Bactris guineensis asistida mediante ultrasonido de sonda directa

Madrid De la Rosa., J.M. Mendoza-Meza., D. y Fontalvo-Gómez., M.

[Kumar et al., 2007] Kumar, A., Kumar, A., y Raheman, H. (2007). Biodiesel production from jatropha oil (Jatropha curcas) with high free fatty acids: An optimized process. Biomass and Bioenergy, 31(8), 569-575. doi: https://doi.org/10.1016/j.biombioe.2007.03.003个Ver página 65

[Lamaisri et al., 2015] Lamaisri, C., Punsuvon, V., Chanprame, S., Arunyanark, A., Srinives, P., y Liangsakul, P. (2015). Relationship between fatty acid composition and biodiesel quality for nine commercial palm oils. Songklanakarin Journal of Science and Technology, 37(4), 389-395. Obtenido de https://www.researchgate.net/publication/281458345_ Relationship_between_fatty_acid_composition_and_biodiesel_quality_for_ nine_commercial_palm_oils $\uparrow$ Ver página 61

[Lee y Saka, 2010] Lee, J., y Saka, S. (2010). Biodiesel production by heterogeneous catalysts and supercritical technologies. Bioresource Technology, 101(19), 7191-7200. doi: https : / / doi . org/10 . $1016 / j$.biortech.2010.04.071 个Ver página 64

[Liu et al., 2018] Liu, S., Chen, W., Zhu, Z., Jiang, S., Ren, T., y Guo, H. (2018). A Review of the Developed New Model Biodiesels and Their Effects on Engine Combustion and Emissions. Applied Sciences, 8(11), 1-16. doi: https://doi .org/10.3390/app8112303 Ver página 53

[Mahamuni y Adewuyi, 2009] Mahamuni, N., y Adewuyi, Y. (2009). Fourier Transform Infrared Spectroscopy (FTIR) Method To Monitor Soy Biodiesel and Soybean Oil in Transesterification Reactions, Petrodiesel-Biodiesel Blends, and Blend Adulteration with Soy Oil. Energy Fuels, 23(7), 3773-3782. doi: https://doi .org/10.1021/ef900130m ^Ver página 67

[Martínez, Arango, y Giraldo, 2019] Martínez, J., Arango, S., y Giraldo, D. (2019). The effects of biofuels on food security: A system dynamics approach for the Colombian case. Sustainable Energy Technologies and Assessments, 34(1), 97-109. doi: https://doi.org/10.1016/j.seta.2019. $05.009 \uparrow$ Ver página 53

[Mejía y Cuty, 2019] Mejía, A., y Cuty, E. (2019). Influencia del biodiésel de aceite de fritura usado, sobre las emisiones: índice de opacidad. Tecnura, 23(59), 60-67. doi: https : / / doi . org/ 10 . $14483 / 22487638.13722 \uparrow$ Ver página 53

[Mendow y Querini, 2016] Mendow, G., y Querini, C. (2016). Argentina Patente $n$ WO2016098025A1. Obtenido de https://patents.google.com/patent/W02016098025A1/un\# patentCitations $\uparrow$ Ver página

[Musa, 2016] Musa, I. (2016). The effects of alcohol to oil molar ratios and the type of alcohol on biodiesel production using transesterification process. Egyptian Journal of Petroleum, 25(1), 21-31. doi: https://doi.org/10.1016/j.ejpe.2015.06.007个Ver página 65 
Producción de biodiesel a partir del aceite extraído de almendra del corozo Bactris guineensis asistida mediante ultrasonido de sonda directa

Madrid De la Rosa., J.M. Mendoza-Meza., D. y Fontalvo-Gómez., M.

[Núñez, 2012] Núñez, D. (2012). Uso de residuos agrícolas para la producción de biocombustibles en el departamento del Meta. Tecnura, 16(34), 142-156. doi: https: / / doi .org/10.14483/ udistrital.jour.tecnura.2012.4.a10个Ver página 53

[Patle et al., 2018] Patle, D. S., Sharma, S., Gadhamsetti, A. P., Balinge, K. R., Bhagat, P. R., Pandit, S., y Kumar, S. (2018). Ultrasonication-Assisted and Benzimidazolium-Based Brønsted Acid Ionic Liquid-Catalyzed Transesterification of Castor Oil. ACS Omega, 3(11), 15455-1546. https : / / doi . org/10.1021/acsomega. 8b02021 个Ver página 54

[Phan y Phan, 2008] Phan, A., y Phan, T. (2008). Biodiesel production from waste cooking oils. Fuel, 87(17-18), 3490-3496. doi: https: / / doi.org/10.1016/j. fuel.2008.07.008 个Ver página 61

[Polo et al., 2018] Polo, L., Fontalvo, M., y Mendoza, D. (2018). Producción de biodiesel mediante transesterificación enzimática de aceite extraído de residuos de la industria de alimentos. Prospectiva, 16(1), 26-33. doi: https: / / doi .org/10.15665/rp.v16i1.1164 个Ver página 54,60

[Pratas et al., 2011] Pratas, M., Freitas, S. D., Oliveira, M., Monteiro, S., Lima, A., y Coutinho, J. (2011). Biodiesel Density: Experimental Measurements and Prediction Models. Energy Fuels, 25(5), 23332340. doi: https://doi.org/10.1021/ef2002124 个Ver página 65

[Rassoulinejad-Mousavi et al., 2018] Rassoulinejad-Mousavi, S., Mao, Y., y Zhang, Y. (2018). Reducing greenhouse gas emissions in Sandia methane-air flame by using a biofuel. Renewable Energy, 128(A), 313-323. doi: https: / / doi.org/10.1016/j.renene.2018.05.079个Ver página 53

[Rivera et al., 2014] Rivera, Y., Gutiérrez, C., Gómez, R., Matute, M., y Izaguirre, C. (2014). Cuantificación del deterioro de aceites vegetales usados en procesos de frituras en establecimientos ubicados en el Municipio Libertador del Estado Mérida. Ciencia e ingeniería, 35(3), 157-164. Obtenido de http://erevistas.saber.ula.ve/index.php/cienciaeingenieria/article/ view/5238/6753 $\uparrow$ Ver página 61

[Rojas, Flórez, y López, 2019] Rojas, A. F., Flórez, C., y López, D. (2019). Prospectivas de aprovechamiento de algunos residuos agro-industriales. Revista Cubana de Química, 31(1), 31-52. Obtenido de http://scielo.sld.cu/scielo.php?script=sci_arttext\&pid= S2224-54212019000100031\&lng=es \&nrm=iso $\uparrow$ Ver página 54

[Romero et al., 2016] Romero, L., Cruz, M., y Sierra, F. (2016). Efecto de la temperatura en el potencial de aprovechamiento energético de los productos de la pirólisis del cuesco de palma. Tecnura, 20(48), 89-99. doi:10.14483/udistrital.jour.tecnura.2016.2.a06 †er página 53

[Ruiz et al., 2016] Ruiz, J., Sandoval, F., y Del Angel, J. (2016). Evaluación del efecto de cantidad de catalizador y relación metanol/aceite para la producción de biodiesel a 
Producción de biodiesel a partir del aceite extraído de almendra del corozo Bactris guineensis asistida mediante ultrasonido de sonda directa

Madrid De la Rosa., J.M. Mendoza-Meza., D. y Fontalvo-Gómez., M.

partir del Cocos nucifera L. Revista de Energía Química y Física, 3(9), 46-55. Obtenido de http://www.ecorfan.org/bolivia/researchjournals/Energia_Quimica_ Y_Fisica/vol3num9/Revista_de_Energia_Quimica_Y_Fisica_V3_N9.pdf $\uparrow$ Ver página 61

[Sahasrabudhe et al., 2017] Sahasrabudhe, S., Rodriguez, V., O'Meara, M., y Farkas, B. (2017). Density, viscosity, and surface tension of five vegetable oils at elevated temperatures: Measurement and modeling. International Journal of Food Properties, 20(S2), 1965-1981. doi: https: / / doi . org/ $10.1080 / 10942912.2017 .1360905 \uparrow$ Ver página 60

[Sahoo y Das, 2009] Sahoo, P., y Das, L. (2009). Process optimization for biodiesel production from Jatropha, Karanja and Polanga oils. Fuel, 88(9), 1588-1594. doi: https : / / doi .org/10 . 1016 / j . fuel.2009.02.016 个Ver página 65

[Stavarache et al., 2006] Stavarache, C., Vinatoru, M., y Maeda, Y. (2006). Ultrasonic versus silent methylation of vegetable oils. Ultrasonics - Sonochemistry, 13(5), 401-407. doi: https : / / doi . org/ $10.1016 / j \cdot u l t$ sonch.2005.08.001 个Ver página 65

[Stavarache et al., 2003] Stavarache, C., Vinatoru, M., Nishimura, R., y Maeda, Y. (2003). Conversion of Vegetable Oil to Biodiesel Using Ultrasonic Irradiation. Chemistry Letters, 32(8), 716-717. doi: https://doi.org/10.1002/chin.200350242个Ver página 61

[Sun et al., 2018] Sun, M., Xu, X., Zhang, Q., Rui, X., Wu, J., y Dong, M. (2018). Ultrasonic-assisted Aqueous Extraction and Physicochemical Characterization of Oil from Clanis bilineata. Journal of Oleo Science, 67(2), 151-165. doi: https:// doi .org/10.5650/jos.ess17108 1 Ver página 64

[Tacias et al., 2016] Tacias, V., Rosales, A., y Torrestiana, B. (2016). Evaluación y caracterización de grasas y aceites residuales de cocina para la producción de biodiésel: un caso de estudio. Revista internacional de contaminación ambiental, 32(3), 303-313. doi: https://doi.org/10.20937/ RICA.2016.32.03.05 个Ver página 60,61

[Teixeira et al., 2018] Teixeira, G., Ávila, S., Silveira, J., Ribani, M., y Ribani, R. (2018). Chemical, thermal and rheological properties and stability of sapucaia (Lecythis pisonis) nut oils. Journal of Thermal Analysis and Calorimetry, 131(3), 2105-2121. doi: https://doi.org/10.1007/ s10973-017-6742-1 个Ver página 53

[Thapa, Indrawan, y Bhoi, 2018] Thapa, S., Indrawan, N., y Bhoi, P. (2018). An overview on fuel properties and prospects of Jatropha biodiesel as fuel for engines. Environmental Technology E Innovation, 9(1), 210-219. doi: https://doi.org/10.1016/j.eti.2017.12.003 †Ver página 53

[Topare et al., 2019] Topare, N., Patil, K., Naik, P., Sonawane, A., y Joshi, P. (2019). Application Ultrasound for Synthesis of Biodiesel. Emerging Trends in Chemical Engineering, 2(1), 1-8. Obteni- 
Producción de biodiesel a partir del aceite extraído de almendra del corozo Bactris guineensis asistida mediante ultrasonido de sonda directa

Madrid De la Rosa., J.M. Mendoza-Meza., D. y Fontalvo-Gómez., M.

do de https://www.researchgate.net/publication/272686765_Application_of_ Ultrasound_for_Synthesis_of_Biodiesel $\uparrow$ Ver página 63

[Torres et al., 2017] Torres, L., Ben-Youseff, C., Alcocer, L., y De la rosa, D. (2017). Efecto de la temperatura y del tiempo de reacción sobre la esterificación y la transesterificación de aceites comestibles usados. Revista de Ciencias Naturales y Agropecuarias, 4(13), 19-35. Obtenido de http://www.ecorfan.org/bolivia/researchjournals/Ciencias_Naturales_y_ Agropecuarias/vol4num13/Revista_de_Ciencias_Naturales_y_Agropecuarias_ V4_N13_3.pdf $\uparrow$ Ver página 63

[Toscano y Maldini, 2007] Toscano, G., y Maldini, E. (2007). Analysis of the physical and chemical characteristics of vegetable oils as fuel. Journal of Agricultural Engineering, 38(3), 39-47. doi: https: //doi.org/10.4081/jae.2007.3.39个Ver página 61

[Veljković et al., 2012] Veljković, V., Avramović, J., y Stamenković, O. (2012). Biodiesel production by ultrasound-assisted transesterification: State of the art and the perspectives. Renewable and Sustainable Energy Reviews, 16(2), 1193-1209. doi: https://doi.org/10.1016/j.rser.2011.11. $022 \uparrow$ Ver página 54,63

[Yu et al., 2017] Yu, G., Nie, J., Lu, L., Wang, S., Li, Z., y Lee, M. (2017). Transesterification of soybean oil by using the synergistic microwave-ultrasonic irradiation. Ultrasonics - Sonochemistry, 39(1), 281-290. doi: https://doi.org/10.1016/j.ultsonch.2017.04.036个Ver página 53

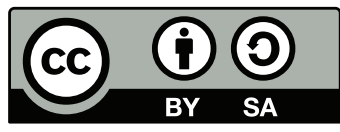

